\title{
A Feasibility Study on Crowdsourcing to Monitor Municipal Resources in Smart Cities
}

\author{
Thivya Kandappu \\ Singapore Management University \\ thivyak@smu.edu.sg
}

\author{
Archan Misra \\ Singapore Management University \\ archanm@smu.edu.sg
}

\author{
Desmond Koh \\ Singapore Management University \\ desmondkoh@smu.edu.sg
}

\author{
Randy Daratan Tandriansyah \\ Singapore Management University \\ rtdaratan@smu.edu.sg
}

\begin{abstract}
Active citizenry, whereby citizens actively participate in reporting and addressing challenges in urban service delivery is a strategic goal of smart cities such as Singapore. In spite of the promise, we believe that the success of such large-scale nation-wide crowdsourcing deployments depend on the real-word user preferences and behavioral characteristics of citizens. In this paper, we first present our findings on behavioral preferences and key concerns of citizens regarding smart-city services via an opinion survey conducted with 1300 participants. We then propose a "citizen-controlled" urban services reporting platform where citizens actively report on the status of various municipal resources. We advocate the importance of matching user mobility patterns against task locations to make the platform more efficient (i.e., higher task completion rate and lower detour overhead).
\end{abstract}

\section{ACM Reference Format:}

Thivya Kandappu, Archan Misra, Desmond Koh, Randy Daratan Tandriansyah, and Nikita Jaiman. 2018. A Feasibility Study on Crowdsourcing to Monitor Municipal Resources in Smart Cities. In WWW' 18 Companion: The 2018 Web Conference Companion, April 23-27, 2018, Lyon, France. ACM, New York, NY, USA, 7 pages. https://doi.org/10.1145/3184558. 3191519

\section{INTRODUCTION}

Mobile crowd-tasking offers an attractive new approach for mobilizing city residents as voluntary participants in the decentralized execution of a variety of smart-city services. Under this paradigm, individual users (or workers) voluntarily select and perform a set of location-specific tasks, such as logistics or package delivery (e.g., Amazon Flex ${ }^{1}$ ), tracking of available parking spaces (e.g., [2]) or gas prices (e.g., [3]) and compliance monitoring/auditing. At present, while there is a significant body of work on computational frameworks for such crowd-tasking (including topics such as optimized

${ }^{1}$ https://flex.amazon.com/

This paper is published under the Creative Commons Attribution 4.0 International (CC BY 4.0) license. Authors reserve their rights to disseminate the work on their personal and corporate Web sites with the appropriate attribution.

WWW' 18 Companion, April 23-27, 2018, Lyon, France

(C) 2018 IW3C2 (International World Wide Web Conference Committee), published under Creative Commons CC BY 4.0 License.

ACM ISBN 978-1-4503-5640-4/18/04.

https://doi.org/10.1145/3184558.3191519

\author{
Nikita Jaiman \\ Singapore Management University \\ nikitaj@smu.edu.sg
}

task allocation, truth discovery and reward specification), real-world embodiments of such large-scale participatory approaches are practically non-existent. In fact, in most cities, residents view the delivery of municipal services as solely the responsibility of the government, and the extent to which such residents would participate as voluntary workers $^{2}$ remains unclear. Obtaining real-time status updates about municipal and urban resources or potential problems with such municipal assets (e.g., overflowing garbage bins, potholes in sidewalks or defaced heritage markers), remains a challenge, especially as the pool of dedicated public employees to perform such status monitoring is fairly limited and overstretched.

With advances in technologies, cities are beginning to automate capture of some of these municipal issues-for example, Pittsburgh's Traffic 21 project $^{3}$ is using cameras mounted on municipal garbage tracks to proactively identify road maintenance issues. Nonetheless, judicious augmentation of such machine-generated insights by trusted, human-generated inputs will remain key for a widelydeployable, cost-effective approach for proactive municipal monitoring.

In the context of urban service delivery, state-of-the-art works usually focus on urban crowd-sourcing for improving local public services $[4,7]$ by receiving feedback/complaints from the citizens. Examples of such Apps include (i) the MySanJose Mobile App ${ }^{4}$ which allows residents to report on issues such as potholes and graffiti, (ii) New York City's NYC311 App ${ }^{5}$ which similarly allows residents to make service requests for potholes, snowy sidewalks and rodent infestation, (iii) Singapore's OneService App ${ }^{6}$ where residents can report about overflowing garbages, abandoned shopping trolleys and pest infestation, and (iv) United Kingdom's FixMyStreet web-App ${ }^{7}$ which enables the public to report problems such as abandoned vehicles and unlit lampposts. Such works exhibit three characteristics: (a) they focus on a "problem reporting" where each crowd-citizen reports issues to the relevant agency, (b) the reporting process is inherently decentralized, with each citizen opportunistically sending one or more complaints that are more relevant to

\footnotetext{
In this paper, we use the terms ŞworkersŤ and ŞusersŤ interchangeably, and they represent citizens of the country.

${ }^{3}$ https://traffic21.heinz.cmu.edu/

${ }^{4}$ http: //www.sanjoseca.gov/MySanJose

${ }^{5}$ http: //www1.nyc.gov/311/index.page

${ }^{6}$ https://www. oneservice.sg

${ }^{7}$ https://www.fixmystreet.com/
} 
him, and (c) the application cannot be used to ask residents to proactively perform checks on the working condition of various municipal resources.

Absent from such approaches are the fundamental concepts of (i) Context-triggered Task Coordination, where the crowd-tasking platform proactively notifies and recommends maintenance-related tasks (compare to the problem reporting fashion) based on the context sensed by other means, such as using social media to sense about faults and repairs (e.g., large number of instagram posts at a location about "clogged drainage"), using historical analytics to learn how susceptible a location is to repairs and natural disasters, and fusing the data from multiple government agencies to understand and predict issues in advance, and (ii) Predictive Crowd-Tasking, where the recommendation or selection of tasks exploits an individual's movement trajectory over a longer time horizon (such as an entire day). Under this concept, residents would be proactively requested to perform various kinds of low-overhead monitoring tasks and upload the status of diverse civic resources. The key novelty lies in the use of analytics technologies, that match tasks and residents mutually based on the task's location and the resident's travel path, such that the resident's detour and effort in performing the task is minimized. Moreover, the application must utilize context awareness to recommend or remind (via appropriate notifications) tasks at opportune moments, when an individual is most likely to actually perform such a monitoring task. We believe that adoption of these two concepts can significantly improve the efficacy of the crowdsourced urban monitoring process.

The end goal is to utilize mobile applications and analytics technologies as a means for embedding participatory crowdsourced tasks in the municipal operations process, as a means to improve the efficiency, reduce the latency and lower the cost of provisioning and delivery of municipal services.

This paper presents (a) a feasibility study conducted with 1300 respondents, checking their receptivity towards smart crowdsourcing effort, and (b) a design of smart urban resources monitoring system and how it leverages both the crowd and mobility to centrally coordinate task distribution among the citizens to increase the task completion rate while lowering the detour overhead incurred. Our key contributions include:

- To assess the willingness of general public to participate in mobile crowdsourcing, we conducted a large-scale opinion survey in our Singapore Management University (SMU) urban campus and reported various factors that could influence users' decision on participating - desired incentive to perform a task, amount of extra time a user is willing to spare, types of tasks the user wants to see and how frequently a user can be alerted about the nearby tasks?

- Based on these insights, we propose a "push-based" mobile crowdsourcing system which incorporates features such as (i) coordinated task recommendations (to reduce potential detour overheads), (ii) localized obfuscation of user reported locations (to assuage privacy concerns), and (iii) contextaware reminders (to improve the participation rate)

In the rest of the paper, we first provide an overview of the survey we conducted and behavioral insights and opinions in $\S 2$. We then provide in-depth details of the proposed urban services monitoring platform Smart City that proactively solicits feedback from citizens in §3. In this section, we describe the role of Smart City mobile App used by citizens, and the backend that consists a location tracking (of citizens) system, an engine that recommends tasks based on the citizens' trajectories, task generator that utilises data from social media and other public sources to identify potential locations that requires frequent reports from the citizens, a privacy engine that masks citizens' locations before revealing them to the Smart City platform, while still able to receive meaningful task recommendations, and a smart task reminder system that notifies citizens about upcoming tasks only at the most opportune moments. Finally, we conclude the paper in $\$ 4$.

\section{SURVEY TO UNDERSTAND ATTITUDES}

In this section, we seek to sample Singapore residents to evaluate the future feasibility of Nation-wide mobile crowdsourcing solutions (a wide-spread approach similar to the Ta\$ker App [6] operational in SMU campus). The main goal is to use opinion surveys to understand the willingness and concerns that Singapore residents may have in getting involved with such crowdsourcing solutions. More specifically, we aim to assess the willingness of general public to participate in mobile crowdsourcing, where, group of people utilise their mobile devices to perform a variety of location sensitive tasks. Examples of such crowdsourced tasks include citizen science (e.g., reporting on whether trees are flowering), and checking of cleanliness/condition of neighbourhood resources (e.g., state land parcels, street lights, heritage markers, parking barriers).

Broadly speaking, our main research question is: "Is the development of a Smart Nation initiative, which is based upon participatory task execution by members of the general public, likely to be successful?" The survey's findings help inform us about the potential adoption issues that the proposed nation-wide crowdsourcing application may face, and thus guide our design of features in this proposed application. For example, analyses of the survey responses might reveal the different parameters that influence the public to perform tasks - such parameters can be: desired incentive to perform a task, amount of extra time a user is willing to spare, types of tasks the user wants to see, how frequently a user can be alerted about the nearby tasks, etc.

\subsection{Demographics of Survey Participants}

The survey was conducted for a 4-week period from 20 March 2017 to 21 April 2017. It attracted around 1300 users (who are part of SMU students and faculty) though only the first 500 respondents were compensated. The survey questionnaire was distributed via email. The overall survey asked each participant to electronically answer a set of questions about his/her opinions and willingness to participate in mobile crowdsourcing by using their mobile devices to perform location specific tasks. More specifically, the survey tackled the following key research themes:

- Is the development of a Smart Nation paradigm, which is based upon participatory task execution by members of the general public, likely to be successful? 


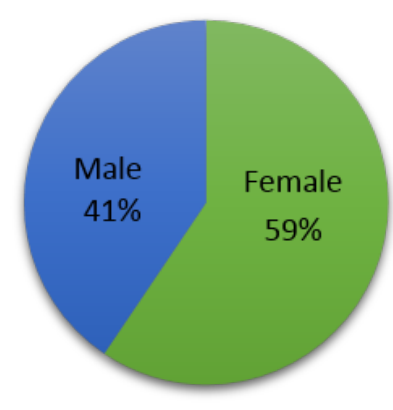

(a) Gender

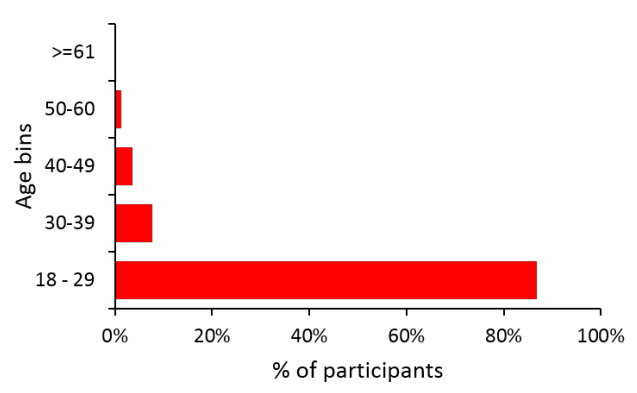

(b) Age

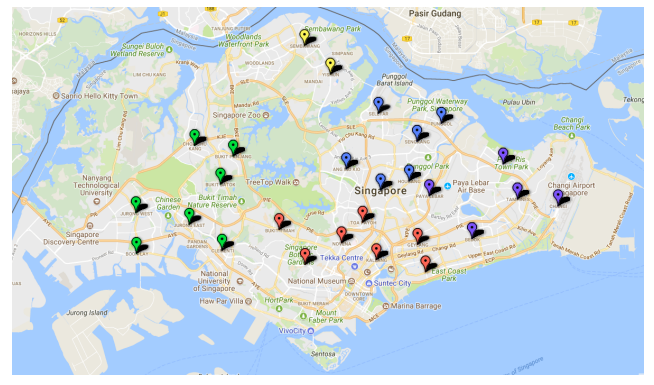

(c) Geographical Distribution

Figure 1: Figures depicting participants demographics: (a) gender, (b) age and (c) geographical residency distribution - coloured markers represent different geographic regions: starting from north of Singapore, clockwise, North, Northeast, East, Central and West regions

- What parameters influence the public to perform tasks - incentive to perform a task, amount of extra time a user is willing to spare, types of tasks the user wants to see, how frequently a user can be alerted about the nearby tasks, etc.?

- What are the concerns that Singapore residents may have in getting involved with such mobile crowdsourcing solutions?

In Fig. 1 we report the basic demographic details. We find that there were significantly more female respondents (59\%) than their male counterparts. Similarly, the majority of the survey respondents were from the age group of 18-29 and lives in the central part (central business district) of Singapore (27\%). We acknowledge the limitation of our study: the majority of the respondents (more than $75 \%$ ) are from SMU's student population and thus not representative of humanity as a whole.

\subsection{Results}

In this section we describe how we address our research questions via the survey questionnaire.

To address our first research question, we asked the survey respondents the following question: How likely are you to provide information (through a mobile App, website, etc.) to improve your living environment?

In Fig. 2 we show the respondents' willingness to participate in crowdtasking paradigm. Regardless of the age group or gender, the majority of the participants appear willing to contribute information to improve their living environment. This suggests that, given a platform with the right features, residents would participate in a smart city initiative that adopts the participatory task execution model.

To address our second question, namely, "what parameters influence the public to perform tasks?", we first ask the respondents about the expected compensation: Do you expect to be incentivised for participating in Crowdtasking?

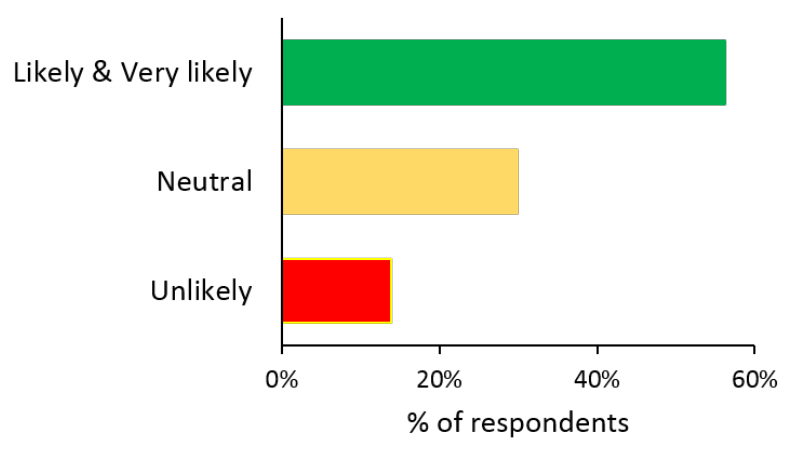

Figure 2: Willingness to participate in mobile crowdsourcing

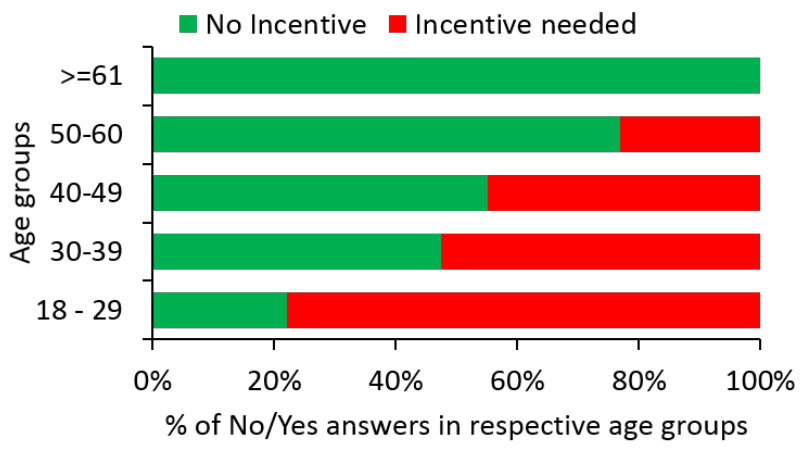

Figure 3: Receptivity for incentives

We find that (in Fig. 3) in general some kind of incentives are expected by significantly larger population - more than $70 \%$ of the respondents expected rewards in cash or in-kind. An interesting trend was observed when we categorize the responses into their respective age groups - majority of the respondents from the younger age 
groups favor some kind of incentives while the older age groups prefer not to incentivized and more specifically they pointed out that "if the task execution is a civic duty or of voluntary in nature, I do not expect to be rewarded" or "I am very active in the field of community service; hence, not in my nature to expect compensation for every community related work that we do".

To see the influence of detour on users' decision to participate in mobile crowdtasking, we asked How much detour are you likely to take, during your daily commute, to help out with such crowdtasking of reports in your living environment?

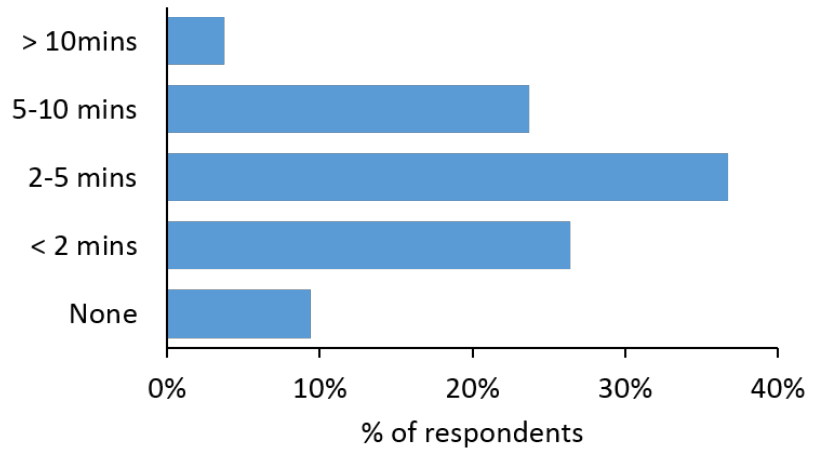

Figure 4: Desired amount of detour

Majority of the users expressed 2-5 minutes of walking distance (equivalent to 100-150 metres) as the desirable detour (refer Fig. 4). This is one critical parameter and needs to be tuned carefully when designing/deploying the system and tasks to the public.

The compelling benefits of the proposed system described in $\S 3$ comes at the cost of user privacy as users' mobility traces and whereabouts are known to a centralised entity. This can be viewed as a breach of privacy by the users, and impact the overall willingness in participating in smart city initiatives. To learn more about user concerns, we asked them What is the main reason for your unwillingness to turn on Location Services?

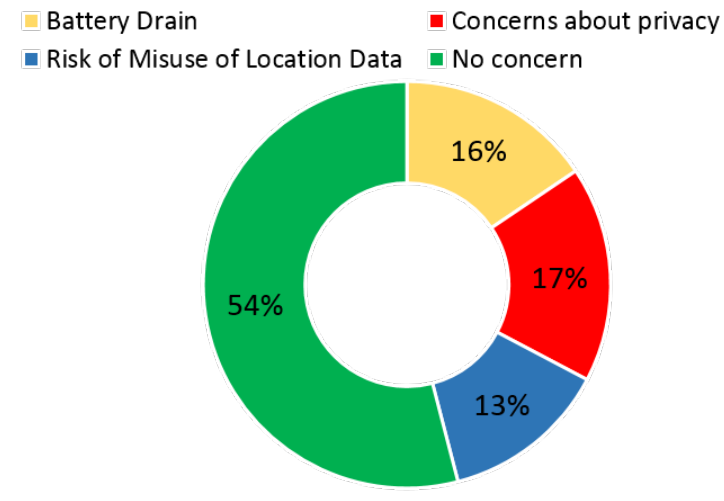

Figure 5: Concerns about location tracking

Surprisingly a significantly larger proportion of the users (more than 50\%) expressed no concerns in being tracked (i.e., collecting location traces) mainly due to the fact that they consider this as their responsibility (refer to Fig. 5). However, a notable set of users expressed their concerns on privacy $(17 \%)$, misuse of location data (13\%) and battery drainage due to continuous GPS polling (15\%).

\subsection{Key Takeaways and Implications:}

- Crowd-powered Smart Monitoring: Exploiting citizens to perform urban monitoring tasks is highly feasible. Their willingness shows that the "power of masses" can be useful to build smart cities by carefully harnessing the citizens.

- Importance of Detour-Aware Recommendations: The surveys show that participant's willingness to perform tasks diminishes quite rapidly with the additional travel overhead. Hence, the platform will require the right kind of intelligence to proactively suggest, to each individual worker, a set of available tasks that do not require substantial deviations from the individual's normal trajectory.

- Variable Pricing: The perception of incentives varies across user age groups. Design of proper incentive mechanisms is thus essential to motivate different groups of users.

- Privacy Support: To address user concerns about location tracking, the system should collect the mobility traces (a) in a privacy preserving manner without revealing the real user locations to anyone (even to the Smart City platform itself), and (b) in an energy efficient way.

\section{MOBILITY AND URBAN CROWDSOURCING}

The platform is aimed at proactively soliciting feedback from the residents on specific municipal resources. In Fig. 6 we depict the overall architecture of our proposed framework. It comprises (a) a client facing mobile App called Smart City, and (b) a backend server that includes a mobility prediction engine, task generator and recommender system.

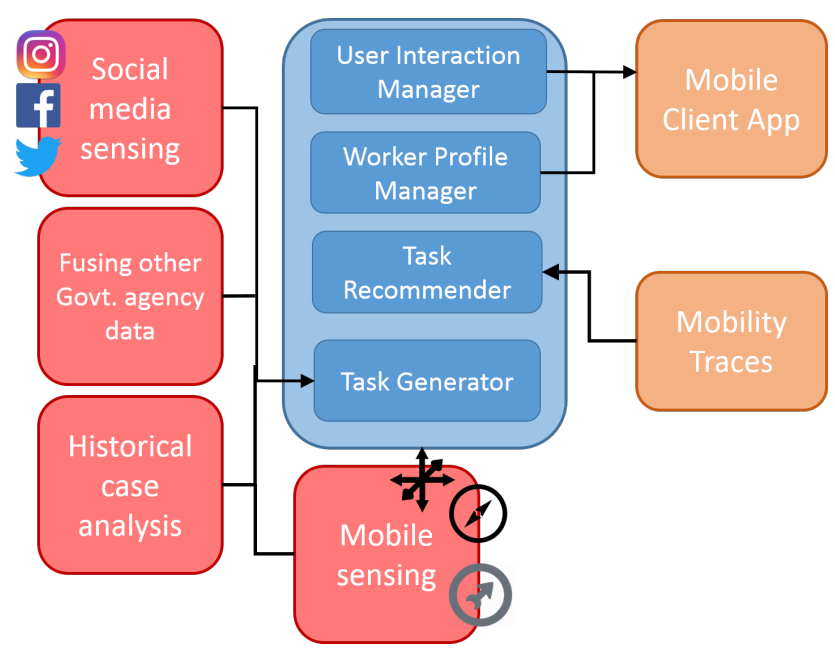

Figure 6: System architecture 


\subsection{User Location Tracking and Predictive Profiling}

A key feature of the push-based approach is the use of predicted individual user trajectories to identify and recommend tasks that maximize the individual user's productivity by allocating tasks that minimize the user's expected travel detour. To activate this component, the Smart City client is augmented to capture the user's GPS-based location trajectory (with the user's consent). The client embeds a mechanism that allows the user to adjust personal filters for such location tracking, for example, by limiting such location tracking to specific time intervals. Using this data, a server-side trajectory prediction engine builds an individual's movement profile (similar to the approach described in $[5,6]$ ), and thereby compute the likely routes of the individual in the near-to-medium future.

\subsection{The Task Recommendation Engine}

This component is the "heart" of push-based mobile crowdsourcing. There are three important categories of information that need to be sent to the recommendation engine: (1) the spatial layout of the city (modeled as a network of location nodes), (2) a set of users (each with a detour time limit, predicted trajectories and preferences), and (3) a set of tasks (each with a location, validity time-window, execution time, and reward). These recommendations utilize advanced geospatial optimization techniques to ensure that both (i) the detour of each individual is reduced significantly, and (ii) the overall set of tasks are adequately "covered" (i.e., assigned to individuals such that there is a high likelihood that the tasks will be successfully executed).

\subsection{Task Generator}

By default, tasks are generated by respective agencies - performing checks on respective municipal resources will be generated as spatiotemporal tasks that will be pulled in to our mobile App Smart City for citizens to use. However, the proliferation of social computing and mobile sensing pave ways for detecting imminent municipal faults and repairs in advance and feeding them as tasks into the App. We shall see three potential scenarios:

- Social media sensing: Social media feeds can be used as spontaneous "human sensors" - a large human population interacts with such media platforms and reports about various events happen in urban spaces, on a daily basis. The exploitation of social feeds can be useful to detect and understand municipal issues such as a tweet about broken street light or an Instagram post on a clogged drainage. Our goals are (1) to detect such municipal issues well in advance by using social media feeds, (2) to actively notify the users who are in the vicinity via a separate channel (a dedicated mobile App) to perform checks and (3) alert the relevant agency about the trouble spot for faster remediation.

In Fig. 7, we show a simple social monitoring pipeline, which gathers geo-tagged feeds from various social networks (such as FaceBook, Twitter and Instagram) to detect topics and (e.g., by using a text classifier on relevant feeds) anomalies (if any). The detected anomalies can be generated as location and time specific tasks in Smart City to proactively notify the users to perform checks (e.g., the public Instagram feeds with the hashtag \#flashfloodsg not only can be used to detect a flash flood but also to understand its severity, by proactively asking the residents in the vicinity).

An early example of such social media sensing based discovery of urban anomalous events is the work in [1], which uses the text and image feeds of Twitter posts to rapidly identify the onset of flash flood events.

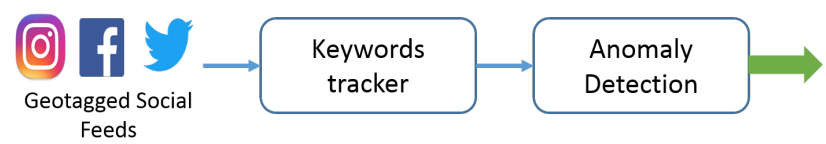

Figure 7: Social Media Sensing to Generate Tasks

- Fusing other agency data: Individual agencies often utilize a dedicated work force to perform the routine monitoring of their relevant municipal resources. Absent from this operating style is coordination across multiple agencies - one agency can leverage a municipal fault detected by other to predict and remediate any resultant issue that would affect it. In Fig. 8, we depict a possible scenario. An upcoming public event (a musical concert in our example) can cause ripples in various sensor readings - the occupancy levels of buses serving the concert location can see a noticeable change well ahead of concert start time (and can be detected by the agency 'A' that maintains and operates buses) while the nearby car parks will see a congestion closer to the event time (deals with the agency ' $\mathrm{B}$ ' that operates public car parks). Agency 'A' informing about the predicted burst in ingress traffic will help the agency ' $\mathrm{B}$ ' to plan and operate car parks efficiently to cater the demand in traffic (e.g., by opening the private car parks to the public).

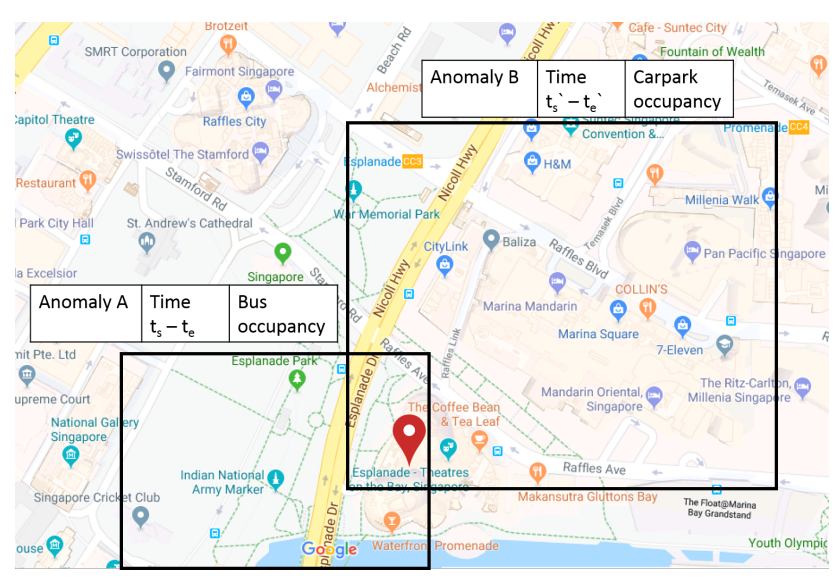

Figure 8: Fusing Multiple Government Agency Data

- Mobile sensing: By efficiently and opportunistically harnessing the in-built sensors of a mobile phone, one can infer various municipal faults - a bumpy/slippery road, damaged 
road surface (potholes), prolonged traffic jams, dangerous driving practices etc. Early detection along with citizen coordination will help the relevant agency to monitor its resources more frequently.

\subsection{Worker Profile Manager}

This server component is responsible for handling interactions of individual workers with back-end components via the Smart City mobile App. Such interactions include providing the App with a list of recommended tasks, capturing the results of the executed tasks (i.e., the values or content reported by the user) and capturing the details of the individual user's interaction with the mobile App (e.g., the time taken to view tasks before selecting them).

\subsection{Privacy Platform}

Despite the promises shown by the proactive recommendation system, the citizens expressed concerns about their location being tracked - around $30 \%$ of the respondents who answered the survey expressed their concerns on the same. To mitigate this privacy issue, we introduce a "user-controlled" obfuscation based location privacy where the client App Smart City obfuscates the users' true location based on (a) global and (b) personal movement behavior. More specifically, the mechanism evaluates the user's sensitivity towards the true location and its global popularity to decide whether to reveal the true location, or obfuscate it with another. This avoids the need of a trusted intermediary and allows them to control the level of obfuscation, without revealing the true location. The client App carefully obfuscates (on behalf of the user) the true location so that the server/platform can not learn accurate details of user whereabouts while still being able to receive task recommendations with lower detour.

\subsection{Context-aware Notifications}

Reminding the users about upcoming or pending tasks is essential to have a steady task accomplishment rate. We can achieve this by sending appropriate notifications via Smart City mobile App. However, interrupting users at the most opportune moment is crucial - though mobile phones made everyone reachable at any time, the utility of interruptions are still questionable. In Smart City, we propose a per-user based context-aware reminder engine that fires in-App notification only when an individual is most likely to actually perform such a monitoring task. The reminder strategy is specifically tailored per user basis based on various contextual attributes, such as his current whereabouts ( a geographical location along with residency period and time of the day will allow us to classify whether he is at work, home or transiting), his activity (by carefully coordinating the accelerometer, compass and gyroscope our engine determines whether the user is walking normally, rushing or running/jogging), collocation of social ties (whether he's accompanied by a friend). Example, the reminder engine refrain itself from sending notifications to a user if the engine detects him rushing (detected based on mobile sensors) from work (longer residency period during the day time and regular arrival/departure patterns suggests his work place) earlier than usual time (time shift in routine trajectory) assuming that the moment is not opportune.

\subsection{Potential Use Cases}

- Matchmaking for community/neighbourhood assistance services: The core technologies proposed in this paper can be embedded in other community-oriented Apps, e.g., to identify available volunteers who can assist the elderly and physicallychallenged neighbors with services, such as grocery assistance and medical visits.

- Crowdsourcing for package delivery: The similar corwdsourcing technology can be used to transport goods or deliver packages by focusing on human-powered mobility. Technologies that support efficient crowdsourced package delivery may be used to involve employees from large-scale public-service organizations (e.g., delivery personnel for postoffice) as workers for various types of monitoring/reporting services.

\section{CONCLUSIONS AND FUTURE WORK}

In this paper, we stress that the success of urban scale smart city deployments depends on behavioral preferences and user concerns. We first showed insights from the large-scale opinion survey we conducted in our urban campus with 1300 participants. Despite the citizens' support/willingness to participate in mobile crowdsourcing based smart-city initiatives, they also expressed concerns on their privacy, possibility of location traces being misused and longer detour tasks. Based on these insights, we proposed a municipal resources monitoring system that leverages the crowd while incorporating features such as (a) proactive task recommendation that matches the user mobility patterns, (b) privacy preserving location sharing, (c) context-aware task reminding, and (d) variable pricing. This gives us hope that given a platform with the right features, residents would participate in a smart city initiative that adopts the participatory task execution model.

\section{ACKNOWLEDGEMENT}

This research is supported by the National Research Foundation, Prime Minister's Office, Singapore under its International Research Centres in Singapore Funding Initiative.

\section{REFERENCES}

[1] Bipendra Basnyat, Amrita Anam abd Neha Singh, and Aryya Gangopadhyayand Nirmalya Roy. 2017. Analyzing Social Media Texts and Images to Assess the Impact of Flash Floods in Cities. In International Conference on Smart Computing (SMARTCOMP).

[2] Xiao Chen, Elizeu Santos-Neto, and Matei Ripeanu. 2012. Crowdsourcing for On-street Smart Parking. In Second ACM International Symposium on Design and Analysis of Intelligent Vehicular Networks and Applications.

[3] Y. F. Dong, S. Kanhere, C. T. Chou, and N. Bulusu. 2008. Automatic Collection of Fuel Prices from a Network of Mobile Cameras.. In 4th IEEE International Conference on Distributed Computing in Sensor Systems.

[4] T. Erickson. 2010. Geocentric crowdsourcing and smarter cities: Enabling urban intelligence in cities and regions. In 1st Ubiquitous Crowdsourcing Workshop at UbiComp.

[5] Thivya Kandappu, Nikita Jaiman, Randy Tandriyansiyah, Archan Misra, Shih-Fen Cheng, Cen Chen, Hoong Chuin Lau, Deepthi Chander, and Koustav Dasgupta. 2016. TASKer: behavioral insights via campus-based experimental mobile crowdsourcing. In 2016 ACM International Joint Conference on Pervasive and Ubiquitous Computing (UbiComp). 
Track: AW4City 2018 Enhancing Citizen

Centricity with Web Applications

WWW 2018, April 23-27, 2018, Lyon, France

[6] Thivya Kandappu, Archan Misra, Shih-Fen Cheng, Nikita Jaiman, Randy Tandriyansiyah, Cen Chen, Hoong Chuin Lau, Deepthi Chander, and Koustav Dasgupta. 2016. Campus-Scale Mobile Crowd-Tasking Deployment \& Behavioral Insights. In The 19th ACM Conference on Computer-Supported Cooperative Work and Social

Computing.

[7] F. Zambonelli. 2010. Pervasive Computing and Communications Workshops (PERCOM Workshops). (2010). 